Proceedings of the Online Conference "Applications of Physics in Mechanical and Material Engineering"

\title{
Frequency-Dependent Chaotic Behavior in RLD Circuit
}

\author{
P. GęBARA ${ }^{a, *}$ AND M. GęBARA ${ }^{b}$ \\ ${ }^{a}$ Department of Physics, Częstochowa University of Technology, \\ al. Armii Krajowej 19, 42-200 Częstochowa, Poland \\ ${ }^{b}$ Office of European Projects, Jan Długosz University in Częstochowa, \\ J. Waszyngtona 4/8, 42-200 Częstochowa, Poland \\ Doi: $10.12693 /$ APhysPolA.139.552 \\ *e-mail: piotr.gebara@pcz.pl
}

\begin{abstract}
The aim of the present paper was to study chaotic behavior in a resistor-inductor diode circuit induced by frequency modulation. Time dependences of voltage and current showed an extremely chaotic response of this system. Based on these dependences, the phase space was built. A bifurcation diagram was constructed and based on it, the Feigenbaum constant was calculated and verified with reliable and noticeable accuracy.
\end{abstract}

topics: deterministic chaos, Feigenbaum constant, phase space

\section{Introduction}

Deterministic chaos is a property of equations or systems of equations, which is related to extremely large sensitivity to initial conditions [1] The simplest example of a chaotic system is a logistic map. Chaotic behavior is observed in many electronic circuits. Period doubling or bifurcation is a simple way to chaos [2,3]. Bifurcations are caused by changing one or several parameters of a system. In the case of a logistic map, the period changes from input 1 to output $2,4,8,16$ and so on. Moreover, the change of the period is realized with a constant step. This constant value $\delta$ is called the Feigenbaum constant [4, 5]. The Feigenbaum diagram is a simple example of a fractal, which means that it is self-similar. A similar structure based on dendritic forms was studied in our previous works [6]. A simple system manifesting chaotic behavior is an RLD circuit, where $\mathrm{R}$ is the resistor, $\mathrm{L}$ - the inductor and $\mathrm{D}$ is the diode (Fig. 1). The diode performs as a nonlinear element.

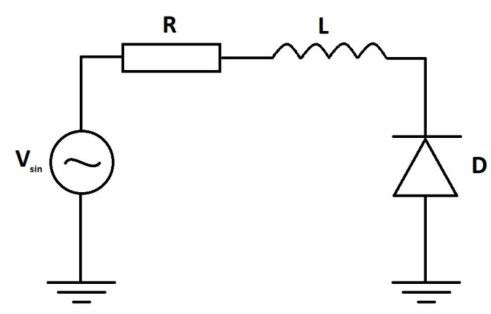

Fig. 1. The scheme of the RLD circuit.
The aim of the present work was to simulate the response of the RLD circuit, construct the Feigenbaum diagram and calculate the Feigenbaum constant.

\section{Simulation procedure}

The simulations were conducted on an RLD circuit using a PC computer (Processor Intel I7, 32GB RAM) equipped with a Wolfram Mathematica package. The simulation was based on the following relations:

$$
I(t)=\frac{\mathrm{d} q(t)}{\mathrm{d} t}
$$

and

$$
\begin{aligned}
& L I^{\prime}(t)+R I(t)+\frac{C_{2}-C_{1}}{2 C_{1} C_{2}}|q(t)| \\
& \quad+\frac{C_{1}+C_{2}}{2 C_{1} C_{2}} q(t)+U_{a}=U_{d} \sin (2 \pi f t),
\end{aligned}
$$

where $I$ is the current, $q$ is the electric charge, $t$ is the time, $L$ is the induction of a solenoid, $R$ is the resistance of a resistor, $C_{1}$ and $C_{2}$ are the capacities of a diode, $U_{a}$ is the voltage, $U_{d}$ is the maximum supply voltage and $f$ is the frequency of a generator.

Implemented in the Mathematica software, (2) was solved numerically using the Runge-Kutta algorithm.

The values of each of the parameters are: $L=0.2 \mathrm{mH}, R=80 \Omega, C_{1}=0.1 \mu \mathrm{F}, C_{2}=60 \mathrm{pF}$, $U_{a}=1.2 \mathrm{~V}$ and $U_{d}=10 \mathrm{~V}$. The parameters of the diode were taken for real diode IN1221. Importantly, the entire procedure was conducted in a wide frequency range. 


\section{Results and discussion}

The time-dependences of voltage for a series of frequencies were depicted in Fig. 2. It is clearly seen that in the beginning, only one period is visible. An increase of frequency induces the multiplying of states and the appearance of a chaotic response of the RLD circuit. The multiplying of the period is clearly seen for frequencies higher than $100 \mathrm{kHz}$.

A more detailed analysis was possible by constructing a phase space. It was plotted as voltage dependences of current in specific values of time and frequencies (Fig. 3). The phase space constructed in a predictable state has an oval shape. The increase of frequency causes the appearance of characteristic loops on the oval shape. Further, the increase of frequency leads to the multiplying of loops, which indicates chaotic behavior.

In order to visualize the further multiplying of the period, the Feigenbaum diagram (FD) was constructed (Fig. 4). The analysis of the FD revealed that for frequencies lower than $30 \mathrm{kHz}$, the circuit generates some ancient states, which is typical for
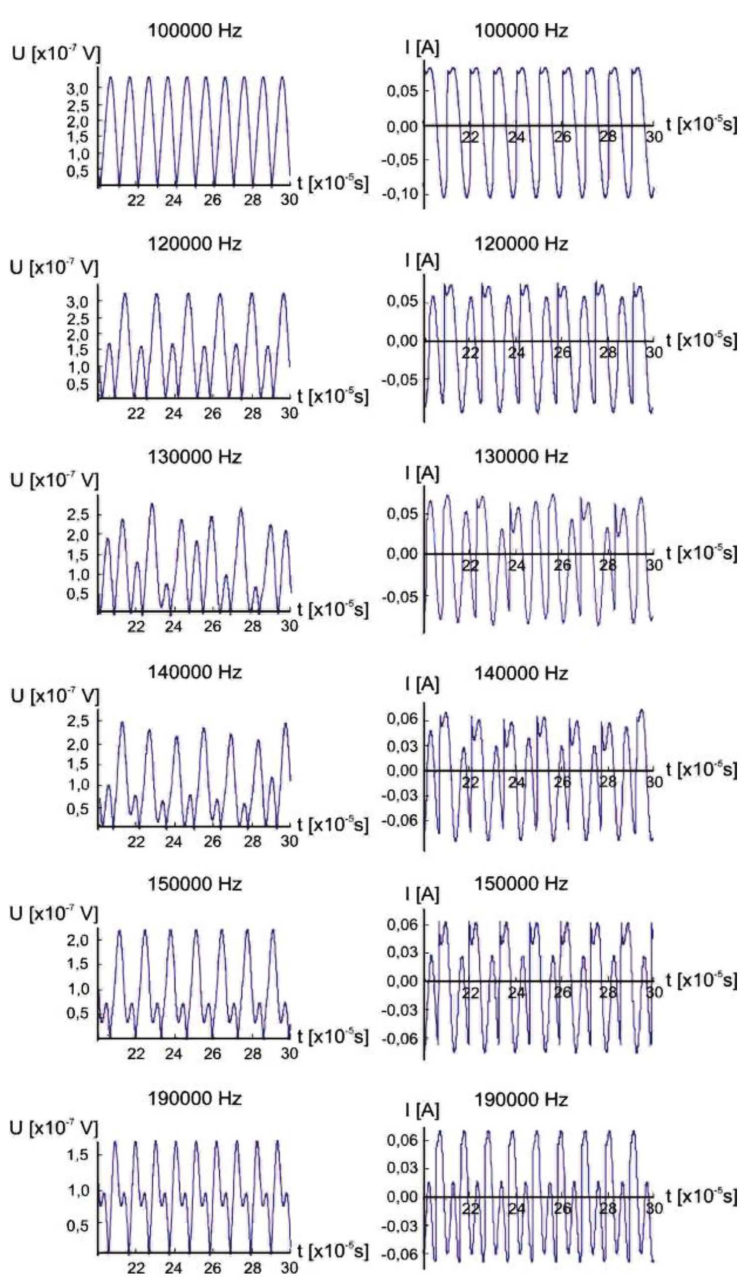

Fig. 2. The time dependences of voltage simulated for different values of frequency for the analyzed RLD circuit.

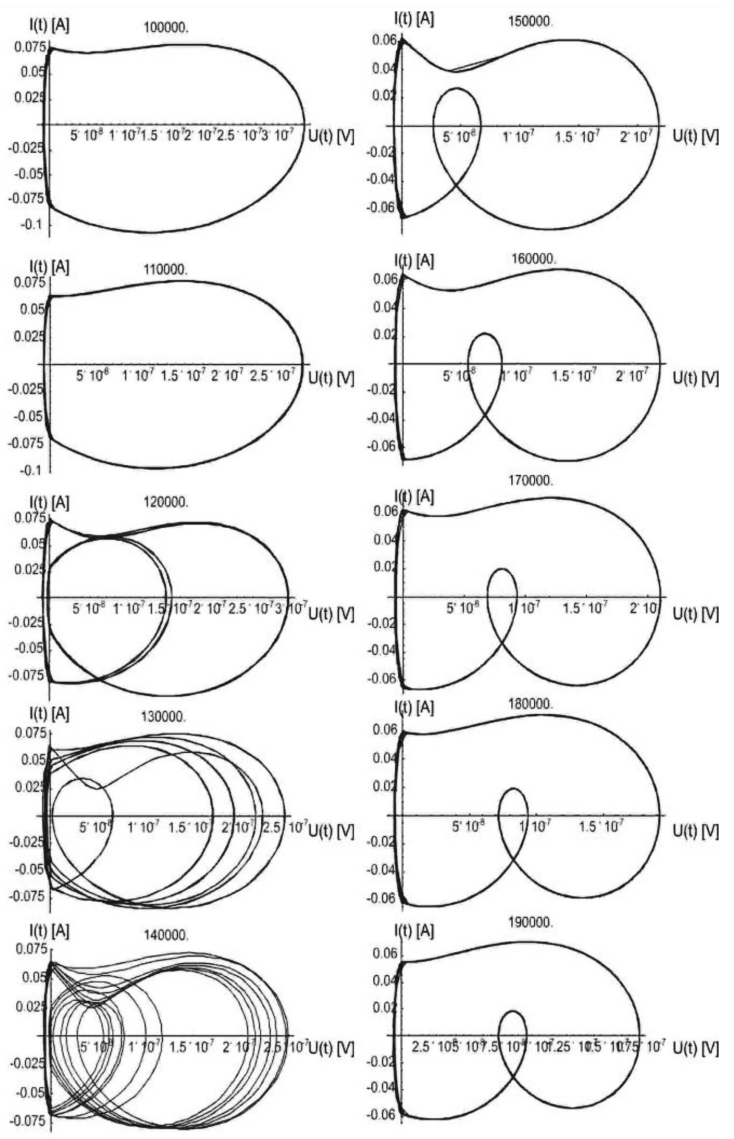

Fig. 3. The phase spaces for different values of frequency for the analyzed RLD circuit.

this type of circuits (i.e., such states are also met in RLC circuits). In the range of $50-100 \mathrm{kHz}$, one period is observed. The doubling of the period was detected for frequencies higher than $130 \mathrm{kHz}$. The next doubling of the period was revealed for the frequency range of $200-220 \mathrm{kHz}$ and then chaotic behavior appeared.

The increase of frequency to $280 \mathrm{kHz}$ caused an additional state and the whole system generated three different states until the next doubling at $325 \mathrm{kHz}$. A similar situation is observed at higher frequencies, when four beginning states were detected. Moreover, it is clearly seen that the amplitude of the period decreases with the increase of frequency. Fitting, used to find a function to the upper border of the diagram, revealed an exponential decrease of the Feigenbaum diagram. Such a behavior could be related to the resistance applied in the studied circuit.

Based on the constructed Feigenbaum diagram, $\delta$ (the Feigenbaum constant) was calculated using [4]

$$
\delta=\frac{x_{n+1}-x_{n}}{x_{n+2}-x_{n+1}},
$$

where $x_{n}$ is the value of the changing parameter with one state and $x_{n+1}$ or $x_{n+2}$ are the parameters corresponding to the next bifurcations. 


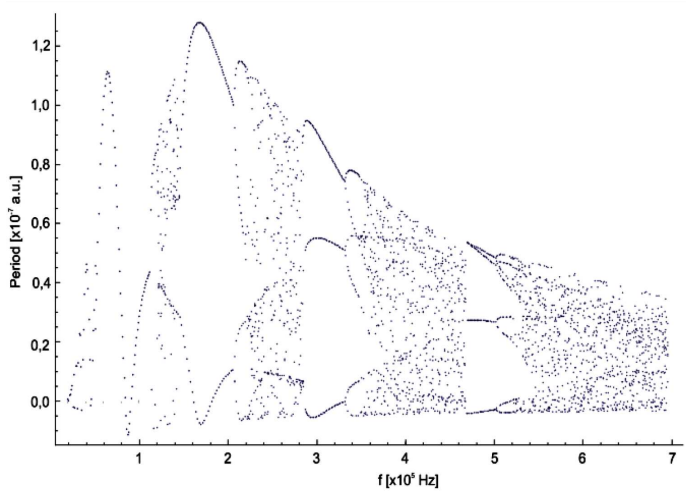

Fig. 4. The Feigenbaum diagram constructed for the analyzed RLD circuit.

In the studied case, (3) should be rewritten in the following way:

$$
\delta=\frac{f_{n+1}-f_{n}}{f_{n+2}-f_{n+1}},
$$

where $f_{n}$ means the changing frequency until the first bifurcation, and $f_{n+1}, f_{n+2}$ are the frequencies to the next two bifurcations.

The calculated Feigenbaum constant equaled $2.85 \pm 0.13$ (for the range of frequencies close to $200 \mathrm{kHz}$ ), $3.72 \pm 0.12$ (for the range of frequencies close to $350 \mathrm{kHz}$ ) and $4.23 \pm 0.12$ (for the range of frequencies close to $500 \mathrm{kHz}$ ) and these values are reliable and reasonable. The theoretical value of the Feigenbaum constant is $4.6692 \ldots$ Taking into account the revealed values, the last one, 4.23 , corresponds well with the theoretical value and a trend to achieve a precise value is visible. Similar values were delivered by Prusha in [7] or Tamasevicius et al. in [8].

A chaotic response of an RLD circuit can potentially be applied to produce one-time pads in secret communication. Each change of some parameter (i.e., the value of induction or resistance) could give a drastically different response in the output, which is extremely important in encoding and decoding.

\section{Conclusions}

In the present paper, the chaotic behavior of the resistor-inductor-diode series circuit was simulated. It was shown that such a simple system is very sensitive to frequency changes. Chaotic behavior was presented using time dependences of the voltage or current. Moreover, it was proven by the construction of the phase space for different values of frequency. Further analysis, including the plotting of the Feigenbaum diagram, confirmed the multiplying of the period with the increase of frequency. This diagram allowed to calculate parameter $\delta$ (the Feigenbaum constant) and it equaled $4.23 \pm 0.12$. Such a value corresponds well with the theoretical predictions.

\section{Acknowledgments}

P.G. would like to thank Professor Jan Świerczek, Head of the Department of Physics, the Częstochowa University of Technology, for financial support.

\section{References}

[1] H.O. Peitgen, H. Jurgens, D. Saupe, Chaos and Fractals, Springer-Verlag, New York 1992.

[2] L. Wang, M. Xu, Chaos Solitons Fract. 19, 527 (2004).

[3] C. Cooper, Chaos Solitons Fract. 24, 157 (2005).

[4] M.J. Feigenbaum, Physica D 7, 16 (1983).

[5] J. San Martin, Chaos Solitons Fract. 32, 816 (2007).

[6] P. Gębara, M. Gębara, A. Owczarek, Acta Phys. Pol. A 138, 287 (2020).

[7] B. Prusha, "Measuring Feigenbaum's $\delta$ in a Bifurcating Electric Circuit" (1997).

[8] A. Tamasevicius, T. Pyragiene, L. Pyragas, S. Bumeliene, M. Meskauskas, Inter. J. Bifurcat. Chaos 17, 3657 (2007). 